\title{
Universal Displacements in Linear Elasticity
}

\author{
Arash Yavari* \\ Christian Goodbrake ${ }^{\dagger}$ \\ Alain Goriely $\ddagger$
}

30 October 2019

\begin{abstract}
In nonlinear elasticity, universal deformations are the deformations that exist for arbitrary strain-energy density functions and suitable tractions at the boundaries. Here, we discuss the equivalent problem for linear elasticity. We characterize the universal displacements of linear elasticity: those displacement fields that can be maintained by applying boundary tractions in the absence of body forces for any linear elastic solid in a given anisotropy class. We show that the universal displacements for compressible isotropic linear elastic solids are constant-divergence harmonic vector fields. We note that any divergence-free displacement field is a universal displacement for incompressible linear elastic solids. Further, we characterize the universal displacement fields for all the anisotropy classes, namely triclinic, monoclinic, tetragonal, trigonal, orthotropic, transversely isotropic, and cubic solids. As expected, universal displacements explicitly depend on the anisotropy class: the smaller the symmetry group, the smaller the space of universal displacements. In the extreme case of triclinic material where the symmetry group only contains the identity and minus identity, the only possible universal displacements are linear homogeneous functions.
\end{abstract}

Keywords: Universal deformation, universal displacement, linear elasticity, anisotropy classes.

\section{Introduction}

A universal deformation in elasticity is one, which is possible in every member of a class of materials in the absence of body forces [Saccomandi, 2001]. In other words, given a class of materials, a universal deformation of a body made of any material in the class is possible by applying only surface tractions.

In the case of (unconstrained) compressible isotropic elastic solids, Ericksen [1955] showed that the only universal deformations are homogeneous deformations. ${ }^{1}$ In [Yavari and Goriely, 2016], we showed that Ericksen's result can be extended to compressible solids with finite eigenstrains that in the absence of eigenstrains are isotropic. In this case, universal deformations in such solids must be covariantly homogeneous. This implies that the material manifold must be flat (assuming a simply-connected body). In other words, universal eigenstrains in compressible isotropic solids are impotent, i.e., are zero-stress.

In the case of incompressible isotropic solids, in addition to homogeneous deformations, Ericksen [1954], Fosdick [1966], Singh and Pipkin [1965] and Klingbeil and Shield [1966] identified the following five families of universal deformations (see Truesdell and Noll [2004], Tadmor, Miller, and Elliott [2012, p.265], and Goriely [2017, p.305] for a visualization and discussion): (1) Bending, stretching, and shearing of a rectangular block; (2) Straightening, stretching, and shearing of a sector of a cylindrical shell; (3) Inflation, bending, torsion, extension, and shearing of a sector of an annular wedge; (4) Inflation/inversion of a sector of a spherical shell; (5) Inflation, bending, extension, and azimuthal shearing of an annular wedge. The possible existence of other families is an open problem in elasticity. These families are limited due to the condition that the deformations must exist for arbitrary isotropic constitutive relationships.

In linear elasticity of simple bodies, the situation is slightly different. For homogeneous solids the constitutive relationships between the stresses and the linear strains is linear with constant coefficients. Hence, we must

\footnotetext{
*School of Civil and Environmental Engineering \& The George W. Woodruff School of Mechanical Engineering, Georgia Institute of Technology, Atlanta, GA 30332, USA. E-mail: arash.yavari@ce.gatech.edu.

${ }^{\dagger}$ Mathematical Institute, University of Oxford, Oxford, OX2 6GG, UK.

${ }^{\ddagger}$ Mathematical Institute, University of Oxford, Oxford, OX2 6GG, UK.

${ }^{1}$ One should note that Ericksen's 1954 and 1955 papers on universal deformations were largely motivated by the earlier works of Rivlin between 1948 and 1954 [Rivlin, 1948, 1949a,b] (see also Truesdell [1952]).
} 
characterize the displacements that exist for arbitrary values of the constant elastic moduli rather than for arbitrary functions. Therefore, we expect a much larger family of solutions and the problem is not to enumerate different possible families of solutions but to characterize fully the set of possible displacements. The question is then: In the absence of body forces, what are the possible universal displacement fields for a homogeneous linear elastic solid? The answer to this question strongly depends on the symmetry group of the material. For instance, we show that for isotropic linear elastic solids any constant-divergence harmonic vector field is a universal displacement field. When the condition of isotropy is relaxed and one considers materials with smaller symmetry groups, we show that the space of universal displacements is further restricted depending on the anisotropy class. We start with a full discussion of the isotropic case and then consider systematically, all the symmetry classes.

\section{Universal displacements in isotropic linear elasticity}

The static equilibrium configuration of a homogeneous and isotropic linear elastic simple body in the absence of body forces is governed by Navier's equation for the displacement vector $\mathbf{u} \in \mathbb{R}^{3}$ :

$$
(\lambda+\mu) \operatorname{grad} \circ \operatorname{div} \mathbf{u}+\mu \Delta \mathbf{u}=\mathbf{0},
$$

where $\lambda$ and $\mu$ are the Lamé constants, and $\Delta \mathbf{u}=\operatorname{div} \circ \operatorname{grad} \mathbf{u}$ is the Laplacian operator. A deformation or displacement field is universal if it can be maintained for all isotropic linear elastic solids by applying only boundary tractions. Navier's equations (2.1) in the absence of body forces can be written as

$$
(1-2 \nu) \Delta \mathbf{u}+\operatorname{grad} \circ \operatorname{div} \mathbf{u}=\mathbf{0},
$$

where $\nu$ is Poisson's ratio. Note that $0<1-2 \nu<3$ for compressible solids. Noting that $\Delta \mathbf{u}=\operatorname{grad} \circ \operatorname{div} \mathbf{u}-$ curl o curl u, Eq. (2.2) can be rewritten as

$$
2(1-\nu) \operatorname{grad} \circ \operatorname{div} \mathbf{u}-(1-2 \nu) \operatorname{curl} \circ \operatorname{curl} \mathbf{u}=\mathbf{0} .
$$

Using (2.2) and (2.3), it follows that $\Delta \Delta \mathbf{u}=\mathbf{0}$, i.e., $\mathbf{u}$ is a biharmonic vector field.

From $(2.2) \mathbf{u}$ is a universal displacement field if (2.2) holds for any $\nu \in\left(-1, \frac{1}{2}\right)$. As $\nu$ is a continuous variable, taking derivative with respect to $\nu$ of both sides of $(2.2)$ one concludes that $-2 \operatorname{grad} \circ \operatorname{div} \mathbf{u}=\mathbf{0}$. Therefore ${ }^{2}$

$$
\operatorname{grad} \circ \operatorname{div} \mathbf{u}=\mathbf{0}, \text { and } \Delta \mathbf{u}=\mathbf{0} .
$$

The first relation implies that $\operatorname{div} \mathbf{u}=c$, where $c$ is a constant, while the second relation shows that $\mathbf{u}$ is a harmonic vector field. Hence, this means that any universal displacement field is a constant-divergence harmonic vector field. Of course, not all (smooth) displacement fields are universal.

On $\mathbb{R}^{3}$ any constant-divergence vector field has the following representation [McLachlan and Quispel, 2002]

$$
u_{a}(x)=S_{a b, b}(x)+\frac{c}{3} x_{a}+k_{a},
$$

where $S_{a b}(x)=-S_{b a}(x)$, and $k_{a}$ are constants. In Cartesian coordinates $(x, y, z)$

$$
\mathbf{S}(x, y, z)=\left[\begin{array}{ccc}
0 & \alpha(x, y, z) & \beta(x, y, z) \\
-\alpha(x, y, z) & 0 & \gamma(x, y, z) \\
-\beta(x, y, z) & -\gamma(x, y, z) & 0
\end{array}\right]
$$

where $\alpha, \beta$, and $\gamma$ are arbitrary functions. Hence

$$
\operatorname{div} \mathbf{S}(x, y, z)=\left[\begin{array}{c}
\alpha_{, y}+\beta_{, z} \\
-\alpha_{, x}+\gamma_{, z} \\
-\beta_{, x}-\gamma_{, y}
\end{array}\right] .
$$

\footnotetext{
${ }^{2}$ These PDE were derived in [Truesdell, 1966]. See also [Gurtin., 1972].
} 
Thus

$$
\Delta \mathbf{u}=\left[\begin{array}{c}
\Delta \alpha_{, y}+\Delta \beta_{, z} \\
\Delta \gamma_{, z}-\Delta \alpha_{, x} \\
-\Delta \beta_{, x}-\Delta \gamma_{, y}
\end{array}\right]
$$

where $\Delta \alpha_{, y}=\alpha_{, y x x}+\alpha_{, y y y}+\alpha_{, y z z}$, and similarly for other terms. Therefore, for a universal displacement field the functions $\alpha, \beta$, and $\gamma$ must satisfy the following system of PDEs

$$
\left\{\begin{array}{l}
\Delta \alpha_{, y}+\Delta \beta_{, z}=0 \\
\Delta \gamma_{, z}-\Delta \alpha_{, x}=0 \\
\Delta \beta_{, x}+\Delta \gamma_{, y}=0
\end{array}\right.
$$

Finding the universal displacements amounts to solving for the functions $\alpha, \beta, \gamma$. Note that $\Delta \alpha, y=(\Delta \alpha), y$, etc. Let $f=\Delta \alpha, g=\Delta \beta$, and $h=\Delta \gamma$. Therefore, we have the following system of linear PDE for the three functions $f, g$, and $h$ :

$$
\left\{\begin{array}{l}
f_{, y}+g_{, z}=0 \\
f_{, x}-h_{, z}=0 \\
g_{, x}+h_{, y}=0 .
\end{array}\right.
$$

This system of PDEs is degenerate. Note that $\left(f_{, x}-h_{, z}\right)_{, y}+\left(g_{, x}+h_{, y}\right)_{, z}=\left(f_{, y}+g_{, z}\right)_{, x}$. Obviously, $f=g=h=0$ is a solution. In this case, $\alpha, \beta$, and $\gamma$ are arbitrary harmonic functions. However, there are many more solutions. Choosing an arbitrary $C^{1}$ function $h(x, y, z)$, from $(2.10)_{2}$ and $(2.10)_{3}, f$ and $g$ are calculated as

$$
\begin{aligned}
& f(x, y, z)=\int h_{, z}(x, y, z) d x+\xi(y, z) \\
& g(x, y, z)=\int-h_{, y}(x, y, z) d x+\eta(y, z) \\
& \xi_{, y}(y, z)+\eta_{, z}(y, z)=0
\end{aligned}
$$

where $\xi$ and $\eta$ are arbitrary $C^{1}$ functions of $y$ and $z$ that satisfy the constraint (2.13). Therefore, in the representation of the universal displacements the functions $(\alpha, \beta, \gamma)$ are the solutions of Poisson's equations $(\Delta \alpha, \Delta \beta, \Delta \gamma)=(f, g, h)$, where $h$ is an arbitrary $C^{1}$ function of $(x, y, z)$, and $\xi$, and $\eta$ are $C^{1}$ functions of $(y, z)$ satisfying the constraint (2.13). Defining the stream function $\psi(y, z)$, and taking $\xi=\frac{\partial \psi}{\partial z}$ and $\eta=-\frac{\partial \psi}{\partial y}$, (2.13) is satisfied, and one obtains

$$
\begin{aligned}
& f(x, y, z)=\int h_{, z}(x, y, z) d x+\frac{\partial \psi(y, z)}{\partial z} \\
& g(x, y, z)=\int-h_{, y}(x, y, z) d x-\frac{\partial \psi(y, z)}{\partial y}
\end{aligned}
$$

Proposition 2.1. For isotropic linear elastic solids all universal displacement fields can be expressed as the superposition of a homogeneous displacement field and a non-homogeneous one, which is the divergence of an anti-symmetric matrix with components that are the solution of Poisson's equation.

Remark 2.2. There have been studies in the literature on the independence of the displacement field in isotropic linear elasticity of either the Poisson's ratio [Carlson, 1971], or the shear modulus [Carlson, 1972]. Universal displacements are different in the sense that they can be maintained in any isotropic linear elastic body in the absence of body forces. However, they may depend on both Poisson's ratio and the shear modulus. In other words, given a universal displacement field in two bodies of the same shape but made of two different isotropic linear elastic solids the boundary tractions needed to maintain the displacement field are different, in general.

Remark 2.3. In the near-incompressible limit, $\lambda$ is a large number, or equivalently $\nu=\frac{1}{2}$. Hence, from $(2.2)$ Navier's equation for incompressible elasticity reads

$$
\operatorname{grad} \circ \operatorname{div} \mathbf{u}=\mathbf{0}
$$

This implies that $\operatorname{div} \mathbf{u}=c$, where $c$ is a constant. Linearization of $J=1$ is $\operatorname{div} \mathbf{u}=0$, and hence $c=0$. It 
is observed that any divergence-free displacement field is a universal displacement field. In other words, all displacement fields that preserve the volume form to the first-order are universal.

\section{Universal displacements in anisotropic linear elasticity}

Next, we consider the eight anisotropy classes (seven classes other than the isotropic class) and find their corresponding universal displacements. In linear elasticity the relation between the Cauchy stress and the linearized strain reads $\sigma_{a b}=c_{a b c d} \epsilon_{c d}$, where $c_{a b c d}$ are the coefficients of the elasticity tensor with major $c_{a b c d}=$ $c_{c d a b}$, and minor symmetries $c_{a b c d}=c_{b a c d}$ (with Roman indices running from 1 to 3 , and summation assumed on repeated indices).

Using the bijection $(11,22,33,23,31,12) \rightarrow(1,2,3,4,5,6)$ the constitutive equations in Voigt notation are written as $\sigma_{\alpha}=c_{\alpha \beta} \epsilon_{\beta}$ (with Greek indices running from 1 to 6 ), where the elasticity tensor is represented by a symmetric $6 \times 6$ stiffness matrix $\mathbf{c}$ :

$$
\mathbf{c}=\left[\begin{array}{llllll}
c_{11} & c_{12} & c_{13} & c_{14} & c_{15} & c_{16} \\
c_{12} & c_{22} & c_{23} & c_{24} & c_{25} & c_{26} \\
c_{13} & c_{23} & c_{33} & c_{34} & c_{35} & c_{36} \\
c_{14} & c_{24} & c_{34} & c_{44} & c_{45} & c_{46} \\
c_{15} & c_{25} & c_{35} & c_{45} & c_{55} & c_{56} \\
c_{16} & c_{26} & c_{36} & c_{46} & c_{56} & c_{66}
\end{array}\right] .
$$

We can further constrain the material class by assuming that the material has some symmetry. These constraints range from the most drastic case of isotropy (with only 2 free constants), to the triclinic case where there is no constraint (with 21 free constants). A useful classification of all possible symmetry group spans these two extreme cases by defining eight symmetry classes depending on the number of symmetry planes, namely: triclinic, monoclinic, tetragonal, trigonal, orthotropic, transversely isotropic, cubic, and isotropic [Cowin and Mehrabadi, 1995; Chadwick, Vianello, and Cowin, 2001; Ting, 2003; Cowin and Doty, 2007], see Fig. 3.1.

\subsection{Universal displacements in triclinic linear elastic solids}

For triclinic solids the identity and its opposite are the only symmetry transformations. For such solids there are no restrictions (other than positive-definiteness) on the elastic constants in (3.1), i.e., there are 21 independent elastic constants. For a body made of a homogeneous anisotropic linear elastic solid, the balance of linear momentum in Cartesian coordinates $\left(x_{1}, x_{2}, x_{3}\right)$ is written as $\sigma_{a b, b}=\left(c_{a b c d} \epsilon_{c d}\right)_{, b}=c_{a b c d} \epsilon_{c d, b}=0$. Using the matrix representation (3.1) this can be rewritten as

$$
\left[\begin{array}{cccccc}
\frac{\partial}{\partial x_{1}} & 0 & 0 & 0 & \frac{\partial}{\partial x_{3}} & \frac{\partial}{\partial x_{2}} \\
0 & \frac{\partial}{\partial x_{2}} & 0 & \frac{\partial}{\partial x_{3}} & 0 & \frac{\partial}{\partial x_{1}} \\
0 & 0 & \frac{\partial}{\partial x_{3}} & \frac{\partial}{\partial x_{2}} & \frac{\partial}{\partial x_{1}} & 0
\end{array}\right]\left[\begin{array}{cccccc}
c_{11} & c_{12} & c_{13} & c_{14} & c_{15} & c_{16} \\
c_{12} & c_{22} & c_{23} & c_{24} & c_{25} & c_{26} \\
c_{13} & c_{23} & c_{33} & c_{34} & c_{35} & c_{36} \\
c_{14} & c_{24} & c_{34} & c_{44} & c_{45} & c_{46} \\
c_{15} & c_{25} & c_{35} & c_{45} & c_{55} & c_{56} \\
c_{16} & c_{26} & c_{36} & c_{46} & c_{56} & c_{66}
\end{array}\right]\left[\begin{array}{c}
\frac{\partial u_{1}}{\partial x_{1}} \\
\frac{\partial u_{2}}{\partial x_{2}} \\
\frac{\partial u_{3}}{\partial x_{3}} \\
\frac{\partial u_{2}}{\partial x_{3}}+\frac{\partial u_{3}}{\partial x_{2}} \\
\frac{\partial u_{1}}{\partial x_{3}}+\frac{\partial u_{3}}{\partial x_{1}} \\
\frac{\partial u_{1}}{\partial x_{2}}+\frac{\partial u_{2}}{\partial x_{1}}
\end{array}\right]=\mathbf{0} .
$$

We note that if we expand these equations explicitly, only fifteen out of twenty one elastic constants enter the equilibrium conditions. The remaining elastic constants enter the tractions. The same issue appears in in other symmetry classes. Nevertheless, the above three equilibrium equations in the absence of body forces should hold for arbitrary values of the independent elastic constants that appear explicitly. This means that in each equilibrium equation the coefficient of each elastic constant should vanish. This gives a set of fifteen PDEs 


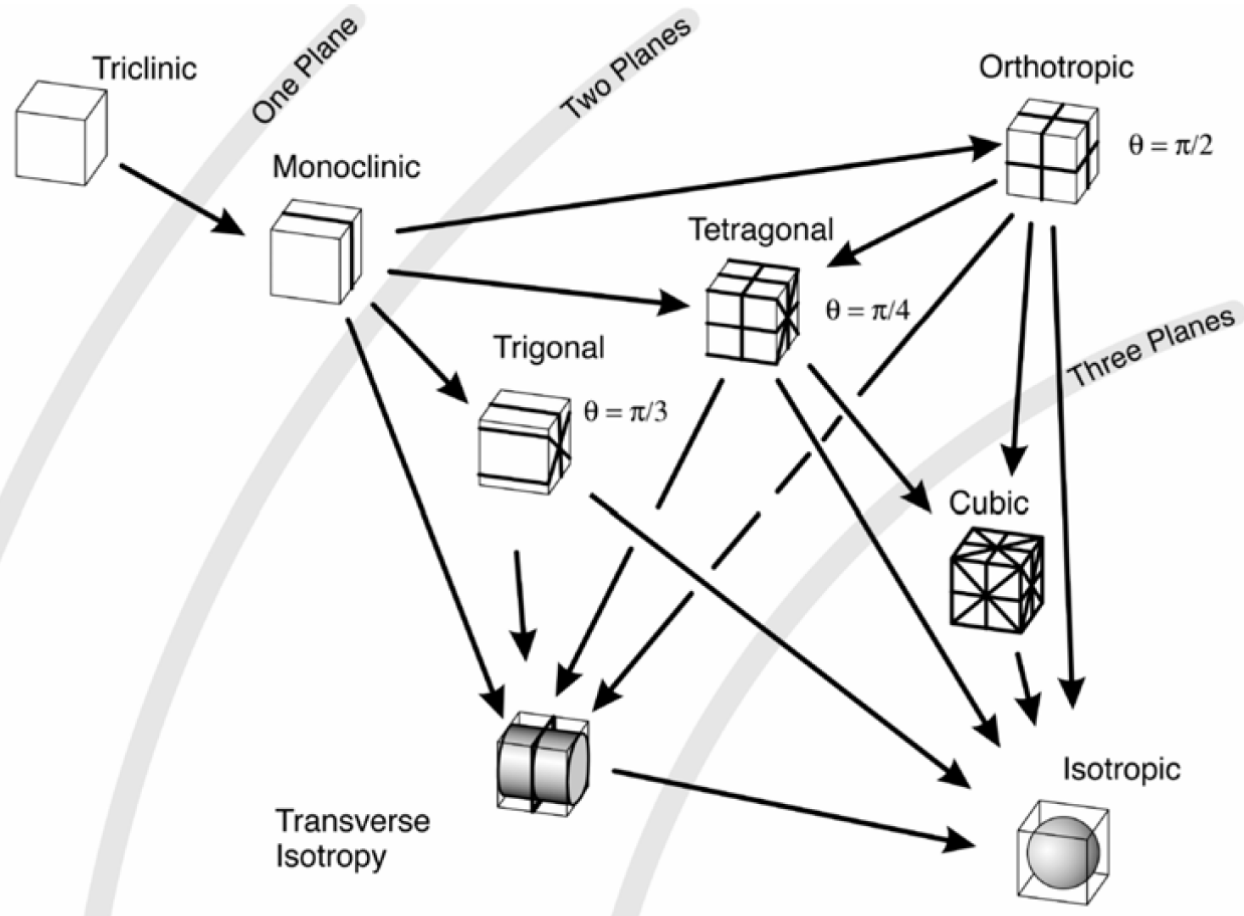

Figure 3.1: The eight material symmetries of linear elasticity. The lower symmetry is the triclinic case and the greater symmetry is the isotropic case. Intermediary cases depend on the number of symmetry planes. With permission from [Chadwick, Vianello, and Cowin, 2001].

for the displacement field. The first set of PDEs are

$$
\begin{aligned}
& \frac{\partial^{2} u_{1}}{\partial x_{1}^{2}}=\frac{\partial^{2} u_{1}}{\partial x_{1} \partial x_{2}}=\frac{\partial^{2} u_{1}}{\partial x_{1} \partial x_{3}}=0 \\
& \frac{\partial^{2} u_{2}}{\partial x_{2}^{2}}=\frac{\partial^{2} u_{2}}{\partial x_{2} \partial x_{1}}=\frac{\partial^{2} u_{2}}{\partial x_{2} \partial x_{3}}=0 \\
& \frac{\partial^{2} u_{3}}{\partial x_{3}^{2}}=\frac{\partial^{2} u_{3}}{\partial x_{3} \partial x_{1}}=\frac{\partial^{2} u_{3}}{\partial x_{2} \partial x_{3}}=0
\end{aligned}
$$

From these equations, one concludes that

$$
\begin{aligned}
& u_{1}\left(x_{1}, x_{2}, x_{3}\right)=c_{1} x_{1}+\hat{u}_{1}\left(x_{2}, x_{3}\right), \\
& u_{2}\left(x_{1}, x_{2}, x_{3}\right)=c_{2} x_{2}+\hat{u}_{2}\left(x_{1}, x_{3}\right), \\
& u_{3}\left(x_{1}, x_{2}, x_{3}\right)=c_{3} x_{3}+\hat{u}_{3}\left(x_{1}, x_{2}\right) .
\end{aligned}
$$

The next set of PDEs is

$$
\frac{\partial^{2} u_{a}}{\partial x_{b}^{2}}=0, \quad a \neq b, a, b \in\{1,2,3\}
$$


The last three PDEs are

$$
\begin{aligned}
& \frac{\partial^{2} u_{1}}{\partial x_{2} \partial x_{3}}=0, \\
& \frac{\partial^{2} u_{2}}{\partial x_{1} \partial x_{3}}=0, \\
& \frac{\partial^{2} u_{3}}{\partial x_{1} \partial x_{2}}=0 .
\end{aligned}
$$

Substituting (3.4) into the above PDE, one obtains

$$
\begin{aligned}
& \hat{u}_{1}\left(x_{2}, x_{3}\right)=f_{1}\left(x_{2}\right)+g_{1}\left(x_{3}\right), \\
& \hat{u}_{2}\left(x_{1}, x_{3}\right)=f_{2}\left(x_{1}\right)+g_{2}\left(x_{3}\right), \\
& \hat{u}_{3}\left(x_{1}, x_{2}\right)=f_{3}\left(x_{1}\right)+g_{3}\left(x_{2}\right) .
\end{aligned}
$$

Substituting the above representations into (3.5) one obtains $f_{a}^{\prime \prime}=0$ and $g_{a}^{\prime \prime}=0, a=1,2,3$, and hence, the displacement field must be homogeneous.

Proposition 3.1. The only universal displacements in triclinic linear elastic solids are homogeneous displacement fields.

\subsection{Universal displacements in monoclinic linear elastic solids}

In a monoclinic solid there is a plane of material symmetry (a reflection symmetry). Without loss of generality, let us assume that $\mathbf{e}_{3}$ is normal to the plane of material symmetry. A monoclinic solid has 13 independent elastic constants, and in the Cartesian coordinates $\left(x_{1}, x_{2}, x_{3}\right)$ the elasticity matrix has the following form:

$$
\mathbf{c}=\left[\begin{array}{cccccc}
c_{11} & c_{12} & c_{13} & 0 & 0 & c_{16} \\
c_{12} & c_{22} & c_{23} & 0 & 0 & c_{26} \\
c_{13} & c_{23} & c_{33} & 0 & 0 & c_{36} \\
0 & 0 & 0 & c_{44} & c_{45} & 0 \\
0 & 0 & 0 & c_{45} & c_{55} & 0 \\
c_{16} & c_{26} & c_{36} & 0 & 0 & c_{66}
\end{array}\right] .
$$

The first set of PDEs governing the displacements is identical to (3.3), and hence, the universal displacements have the form (3.4). The second set of PDEs in terms of $\hat{u}_{a}$ read

$$
\begin{aligned}
& \frac{\partial^{2} \hat{u}_{1}}{\partial x_{2}^{2}}=\frac{\partial^{2} \hat{u}_{1}}{\partial x_{3}^{2}}=0, \\
& \frac{\partial^{2} \hat{u}_{2}}{\partial x_{1}^{2}}=\frac{\partial^{2} \hat{u}_{2}}{\partial x_{3}^{2}}=0, \\
& \frac{\partial^{2} \hat{u}_{3}}{\partial x_{1}^{2}}=\frac{\partial^{2} \hat{u}_{3}}{\partial x_{2}^{2}}=0 .
\end{aligned}
$$

The last set of PDEs in terms of $\hat{u}_{a}$ read

$$
\begin{aligned}
& \frac{\partial^{2} \hat{u}_{1}}{\partial x_{2} \partial x_{3}}+\frac{\partial^{2} \hat{u}_{2}}{\partial x_{1} \partial x_{3}}=0, \\
& \frac{\partial^{2} \hat{u}_{3}}{\partial x_{1} \partial x_{2}}=0 .
\end{aligned}
$$

From (3.15) one concludes that

$$
\hat{u}_{3}\left(x_{1}, x_{2}\right)=F_{3}\left(x_{1}\right)+G_{3}\left(x_{2}\right),
$$

and from (3.13) we have $F_{3}^{\prime \prime}\left(x_{1}\right)=G_{3}^{\prime \prime}\left(x_{2}\right)=0$. This means that $u_{3}$ is a homogeneous displacement component. From (3.11) and (3.12) one obtains $u_{1}\left(x_{1}, x_{2}, x_{3}\right)=u_{1}\left(x_{1}, x_{2}, x_{3}\right)^{\text {hom }}+a_{1} x_{2} x_{3}$, and $u_{2}\left(x_{1}, x_{2}, x_{3}\right)=$ 
$u_{2}\left(x_{1}, x_{2}, x_{3}\right)^{\text {hom }}+a_{2} x_{1} x_{3}$. Finally, (3.14) dictates that $a_{1}+a_{2}=0$. Thus, we have the following characterization of the universal displacements.

Proposition 3.2. The universal displacements in a monoclinic linear elastic solid with planes of symmetry parallel to the $x_{1} x_{2}$-plane are the superposition of homogeneous displacement fields and the one-parameter inhomogeneous displacement field $\left(c x_{2} x_{3},-c x_{1} x_{3}, 0\right)$.

\subsection{Universal displacements in tetragonal linear elastic solids}

A tetragonal solid has five planes of symmetry, normals of four of which are coplanar while the fifth one is normal to the other four. Without loss of generality, we assume that in the Cartesian coordinate system $\left(x_{1}, x_{2}, x_{3}\right)$ the fifth normal is along the $x_{3}$ axis (with its corresponding plane of symmetry parallel to the $x_{1} x_{2}$-plane). The first two planes of symmetry are parallel to the $x_{1} x_{3}$ and $x_{2} x_{3}$-planes. The other two planes of symmetry are related to the ones parallel to the $x_{1} x_{3}$-plane by $\pi / 4$ and $3 \pi / 4$ rotations about the $x_{3}$ axis. A tetragonal solid has 6 independent elastic constants, and in the Cartesian coordinates $\left(x_{1}, x_{2}, x_{3}\right)$ the symmetric elasticity matrix has the following form:

$$
\mathbf{c}=\left[\begin{array}{cccccc}
c_{11} & c_{12} & c_{13} & 0 & 0 & 0 \\
c_{12} & c_{11} & c_{13} & 0 & 0 & 0 \\
c_{13} & c_{13} & c_{33} & 0 & 0 & 0 \\
0 & 0 & 0 & c_{44} & 0 & 0 \\
0 & 0 & 0 & 0 & c_{44} & 0 \\
0 & 0 & 0 & 0 & 0 & c_{66}
\end{array}\right] .
$$

Using this representation in the Navier's equations and assuming the arbitrariness of the six elastic constants result in the following PDEs for the universal displacements.

$$
\begin{aligned}
& \frac{\partial^{2} u_{1}}{\partial x_{1}^{2}}=\frac{\partial^{2} u_{1}}{\partial x_{1} \partial x_{2}}=\frac{\partial^{2} u_{1}}{\partial x_{2}^{2}}=\frac{\partial^{2} u_{1}}{\partial x_{3}^{2}}=0, \\
& \frac{\partial^{2} u_{2}}{\partial x_{1} \partial x_{2}}=\frac{\partial^{2} u_{2}}{\partial x_{2}^{2}}=\frac{\partial^{2} u_{2}}{\partial x_{1}^{2}}=\frac{\partial^{2} u_{2}}{\partial x_{3}^{2}}=0, \\
& \frac{\partial^{2} u_{1}}{\partial x_{1} \partial x_{3}}+\frac{\partial^{2} u_{2}}{\partial x_{2} \partial x_{3}}=0, \\
& \frac{\partial^{2} u_{3}}{\partial x_{1} \partial x_{3}}=\frac{\partial^{2} u_{3}}{\partial x_{2} \partial x_{3}}=\frac{\partial^{2} u_{3}}{\partial x_{3}^{2}}=0, \\
& \frac{\partial^{2} u_{3}}{\partial x_{1}^{2}}+\frac{\partial^{2} u_{3}}{\partial x_{2}^{2}}=0 .
\end{aligned}
$$

We note that since general homogeneous displacements exist for the triclinic case, they exist for all linear elastic solids. Hence, we focus on finding the inhomogeneous solutions. From (3.21) and (3.22) one concludes that

$$
u_{3}\left(x_{1}, x_{2}, x_{3}\right)=c_{3} x_{3}+\hat{u}_{3}\left(x_{1}, x_{2}\right), \quad \nabla^{2} \hat{u}_{3}=\frac{\partial^{2} \hat{u}_{3}}{\partial x_{1}^{2}}+\frac{\partial^{2} \hat{u}_{3}}{\partial x_{2}^{2}}=0 .
$$

From (3.18)-(3.20) one concludes that the inhomogeneous part of $u_{1}$ is of the form $c_{1} x_{2} x_{3}+c_{2} x_{1} x_{3}$, and that of $u_{2}$ is of the form $-c_{2} x_{2} x_{3}+c_{3} x_{1} x_{3}$. Therefore, we have the following characterization of universal displacements.

Proposition 3.3. The universal displacements in a tetragonal linear elastic solid with the tetragonal axes parallel to the $x_{3}$-axis in a Cartesian coordinate system $\left(x_{1}, x_{2}, x_{3}\right)$ are the superposition of homogeneous displacement fields and the following inhomogeneous displacement field:

$$
\begin{aligned}
& u_{1}^{\text {inh }}\left(x_{1}, x_{2}, x_{3}\right)=c_{1} x_{2} x_{3}+c_{2} x_{1} x_{3}, \\
& u_{2}^{\text {inh }}\left(x_{1}, x_{2}, x_{3}\right)=-c_{2} x_{1} x_{3}+c_{3} x_{1} x_{3}, \\
& u_{3}^{\text {inh }}\left(x_{1}, x_{2}, x_{3}\right)=g\left(x_{1}, x_{2}\right),
\end{aligned}
$$

where $c_{1}$ and $c_{2}$ are constants, and $g=g\left(x_{1}, x_{2}\right)$ is a harmonic function. 


\subsection{Universal displacements in trigonal linear elastic solids}

A trigonal solid has three planes of symmetry with normals that lie in the same plane and are related by $\pi / 3$ rotations. In other words, two of the planes of symmetry are related to the third one by rotations about a fixed axis by $\pi / 3$ and $-\pi / 3$. In a Cartesian coordinate system $\left(x_{1}, x_{2}, x_{3}\right)$ let us assume that the trigonal axis is the $x_{3}$-axis. A trigonal solid has 6 independent elastic constants, and in the Cartesian coordinates $\left(x_{1}, x_{2}, x_{3}\right)$ its elasticity matrix has the following representation:

$$
\mathbf{c}=\left[\begin{array}{cccccc}
c_{11} & c_{12} & c_{13} & 0 & c_{15} & 0 \\
c_{12} & c_{11} & c_{13} & 0 & -c_{15} & 0 \\
c_{13} & c_{13} & c_{33} & 0 & 0 & 0 \\
0 & 0 & 0 & c_{44} & 0 & -c_{15} \\
c_{15} & -c_{15} & 0 & 0 & c_{44} & 0 \\
0 & 0 & 0 & -c_{15} & 0 & \frac{1}{2}\left(c_{11}-c_{12}\right)
\end{array}\right] .
$$

Again, Navier's equations and the arbitrariness of the six elastic constants give the following PDEs for the universal displacements.

$$
\begin{aligned}
& \frac{\partial^{2} u_{3}}{\partial x_{1} \partial x_{3}}=\frac{\partial^{2} u_{3}}{\partial x_{2} \partial x_{3}}=\frac{\partial^{2} u_{3}}{\partial x_{3}^{2}}=0 \\
& \frac{\partial^{2} u_{1}}{\partial x_{1}^{2}}+\frac{\partial^{2} u_{1}}{\partial x_{2}^{2}}=\frac{\partial^{2} u_{2}}{\partial x_{1}^{2}}+\frac{\partial^{2} u_{2}}{\partial x_{2}^{2}}=\frac{\partial^{2} u_{3}}{\partial x_{1}^{2}}+\frac{\partial^{2} u_{3}}{\partial x_{2}^{2}}=0 \\
& \frac{\partial^{2} u_{1}}{\partial x_{3}^{2}}=\frac{\partial^{2} u_{2}}{\partial x_{3}^{2}}=0, \\
& \frac{\partial^{2} u_{1}}{\partial x_{1} \partial x_{2}}=\frac{\partial^{2} u_{2}}{\partial x_{1}^{2}}, \quad \frac{\partial^{2} u_{2}}{\partial x_{1} \partial x_{2}}=\frac{\partial^{2} u_{1}}{\partial x_{2}^{2}} \\
& 2 \frac{\partial^{2} u_{1}}{\partial x_{1} \partial x_{3}}-2 \frac{\partial^{2} u_{2}}{\partial x_{2} \partial x_{3}}+\frac{\partial^{2} u_{3}}{\partial x_{1}^{2}}-\frac{\partial^{2} u_{3}}{\partial x_{2}^{2}}=0 \\
& \frac{\partial^{2} u_{1}}{\partial x_{2} \partial x_{3}}+\frac{\partial^{2} u_{2}}{\partial x_{1} \partial x_{3}}+\frac{\partial^{2} u_{3}}{\partial x_{1} \partial x_{2}}=0 \\
& \frac{\partial^{2} u_{1}}{\partial x_{1} \partial x_{3}}+\frac{\partial^{2} u_{2}}{\partial x_{2} \partial x_{3}}=0 \\
& \frac{\partial^{2} u_{1}}{\partial x_{1}^{2}}=3 \frac{\partial^{2} u_{1}}{\partial x_{2}^{2}} \cdot
\end{aligned}
$$

From $(3.28)$ and $(3.29)_{3}$ one concludes that

$$
u_{3}\left(x_{1}, x_{2}, x_{3}\right)=c_{3} x_{3}+\hat{u}_{3}\left(x_{1}, x_{2}\right), \quad \nabla^{2} \hat{u}_{3}=\frac{\partial^{2} \hat{u}_{3}}{\partial x_{1}^{2}}+\frac{\partial^{2} \hat{u}_{3}}{\partial x_{2}^{2}}=0 .
$$

From (3.35) and $(3.29)_{1}$ we have

$$
\frac{\partial^{2} u_{1}}{\partial x_{1}^{2}}=\frac{\partial^{2} u_{1}}{\partial x_{2}^{2}}=0
$$

From (3.37) and $(3.30)_{1}$ one concludes that

$$
u_{1}\left(x_{1}, x_{2}, x_{3}\right)=a_{123} x_{1} x_{2} x_{3}+a_{12} x_{1} x_{2}+a_{13} x_{1} x_{3}+a_{23} x_{2} x_{3}+a_{1} x_{1}+a_{2} x_{2}+a_{3} x_{3}+a_{0} .
$$

From $(3.30)_{2}$ one concludes that

$$
u_{2}\left(x_{1}, x_{2}, x_{3}\right)=x_{3} f\left(x_{1}, x_{2}\right)+g\left(x_{1}, x_{2}\right) \text {. }
$$


Using (3.37) and (3.31) $)_{2}$ we have $\frac{\partial^{2} u_{2}}{\partial x_{1} \partial x_{2}}=0$, and hence, $\frac{\partial^{2} f}{\partial x_{1} \partial x_{2}}=\frac{\partial^{2} g}{\partial x_{1} \partial x_{2}}=0$. Thus

$$
u_{2}\left(x_{1}, x_{2}, x_{3}\right)=x_{3}\left[f_{1}\left(x_{1}\right)+f_{2}\left(x_{2}\right)\right]+g_{1}\left(x_{1}\right)+g_{2}\left(x_{2}\right) .
$$

From $(3.29)_{2}$

$$
f_{1}^{\prime \prime}\left(x_{1}\right)+f_{2}^{\prime \prime}\left(x_{2}\right)=g_{1}^{\prime \prime}\left(x_{1}\right)+g_{2}^{\prime \prime}\left(x_{2}\right)=0 .
$$

Thus, $f_{1}^{\prime \prime}\left(x_{1}\right)=-f_{2}^{\prime \prime}\left(x_{2}\right)=a$, and $g_{1}^{\prime \prime}\left(x_{1}\right)=-g_{2}^{\prime \prime}\left(x_{2}\right)=\bar{a}$. Therefore

$$
u_{2}\left(x_{1}, x_{2}, x_{3}\right)=\frac{1}{2}\left(\bar{a}+a x_{3}\right)\left(x_{1}^{2}-x_{2}^{2}\right)+b_{13} x_{1} x_{3}+b_{23} x_{2} x_{3}+b_{1} x_{1}+b_{2} x_{2}+b_{3} x_{3}+b_{0} .
$$

Substituting (3.38) and (3.42) into (3.34): $a_{13}+b_{23}+\left(a_{123}-a\right) x_{2}=0$, which gives $a=a_{123}$, and $b_{23}=-a_{13}$. Also, from $(3.31)_{1}, \bar{a}=a_{12}$. Thus

$$
u_{2}\left(x_{1}, x_{2}, x_{3}\right)=\frac{1}{2}\left(a_{12}+a_{123} x_{3}\right)\left(x_{1}^{2}-x_{2}^{2}\right)+b_{13} x_{1} x_{3}-a_{13} x_{2} x_{3}+b_{1} x_{1}+b_{2} x_{2}+b_{3} x_{3}+b_{0} .
$$

From (3.33): $\frac{\partial^{2} \hat{u}_{3}}{\partial x_{1} \partial x_{2}}=-2 a_{123} x_{1}-\left(a_{23}+b_{13}\right)$, and hence

$$
\hat{u}_{3}\left(x_{1}, x_{2}\right)=-a_{123} x_{1}^{2} x_{2}-\left(a_{23}+b_{13}\right) x_{1} x_{2}+f\left(x_{1}\right)+g\left(x_{2}\right) .
$$

From $(3.36)_{2}$, one obtains

$$
f^{\prime \prime}\left(x_{1}\right)+g^{\prime \prime}\left(x_{2}\right)=2 a_{123} x_{2} .
$$

Thus

$$
u_{3}\left(x_{1}, x_{2}, x_{3}\right)=-a_{123} x_{1}^{2} x_{2}-\left(a_{23}+b_{13}\right) x_{1} x_{2}+\frac{1}{3} a_{123} x_{2}^{3}+\frac{1}{2} c\left(x_{1}^{2}-x_{2}^{2}\right)+c_{1} x_{1}+c_{2} x_{2}+c_{3} x_{3}+c_{0} .
$$

Finally, substituting (3.38), (3.43), and (3.46) into (3.32), one gets $c=-2 a_{13}$. Therefore, we have the following characterization of the universal deformations.

Proposition 3.4. The universal displacements in a trigonal linear elastic solid are the superposition of homogeneous displacements and the following inhomogeneous displacement fields:

$$
\begin{aligned}
& u_{1}^{i n h}\left(x_{1}, x_{2}, x_{3}\right)=a_{123} x_{1} x_{2} x_{3}+a_{12} x_{1} x_{2}+a_{13} x_{1} x_{3}+a_{23} x_{2} x_{3}, \\
& u_{2}^{i n h}\left(x_{1}, x_{2}, x_{3}\right)=\frac{1}{2}\left(a_{12}+a_{123} x_{3}\right)\left(x_{1}^{2}-x_{2}^{2}\right)+b_{13} x_{1} x_{3}-a_{13} x_{2} x_{3}, \\
& u_{3}^{i n h}\left(x_{1}, x_{2}, x_{3}\right)=-a_{123} x_{1}^{2} x_{2}-\left(a_{23}+b_{13}\right) x_{1} x_{2}+\frac{1}{3} a_{123} x_{2}^{3}-a_{13}\left(x_{1}^{2}-x_{2}^{2}\right) .
\end{aligned}
$$

\subsection{Universal displacements in orthotropic linear elastic solids}

An orthotropic solid has three mutually orthogonal symmetry planes. Let us assume that these are the coordinate planes in the Cartesian coordinates $\left(x_{1}, x_{2}, x_{3}\right)$. An orthotropic solid has 9 independent elastic constants, and in the Cartesian coordinates $\left(x_{1}, x_{2}, x_{3}\right)$ its elasticity matrix has the following representation:

$$
\mathbf{c}=\left[\begin{array}{cccccc}
c_{11} & c_{12} & c_{13} & 0 & 0 & 0 \\
c_{12} & c_{22} & c_{23} & 0 & 0 & 0 \\
c_{13} & c_{23} & c_{33} & 0 & 0 & 0 \\
0 & 0 & 0 & c_{44} & 0 & 0 \\
0 & 0 & 0 & 0 & c_{55} & 0 \\
0 & 0 & 0 & 0 & 0 & c_{66}
\end{array}\right] .
$$


Navier's equations and arbitrariness of the nine elastic constants result in the following PDEs for the universal displacements.

$$
\begin{aligned}
\frac{\partial^{2} u_{1}}{\partial x_{1}^{2}} & =\frac{\partial^{2} u_{1}}{\partial x_{1} \partial x_{2}}=\frac{\partial^{2} u_{1}}{\partial x_{1} \partial x_{3}}=0, \\
\frac{\partial^{2} u_{2}}{\partial x_{2}^{2}} & =\frac{\partial^{2} u_{2}}{\partial x_{2} \partial x_{1}}=\frac{\partial^{2} u_{2}}{\partial x_{2} \partial x_{3}}=0, \\
\frac{\partial^{2} u_{3}}{\partial x_{3}^{2}} & =\frac{\partial^{2} u_{3}}{\partial x_{3} \partial x_{1}}=\frac{\partial^{2} u_{3}}{\partial x_{2} \partial x_{3}}=0, \\
\frac{\partial^{2} u_{1}}{\partial x_{2}^{2}} & =\frac{\partial^{2} u_{1}}{\partial x_{3}^{2}}=0 \\
\frac{\partial^{2} u_{2}}{\partial x_{1}^{2}} & =\frac{\partial^{2} u_{2}}{\partial x_{3}^{2}}=0 \\
\frac{\partial^{2} u_{3}}{\partial x_{1}^{2}} & =\frac{\partial^{2} u_{3}}{\partial x_{2}^{2}}=0 .
\end{aligned}
$$

From (3.51)-(3.53) one concludes that

$$
\begin{aligned}
& u_{1}\left(x_{1}, x_{2}, x_{3}\right)=c_{1} x_{1}+\hat{u}_{1}\left(x_{2}, x_{3}\right), \\
& u_{2}\left(x_{1}, x_{2}, x_{3}\right)=c_{2} x_{2}+\hat{u}_{2}\left(x_{1}, x_{3}\right), \\
& u_{3}\left(x_{1}, x_{2}, x_{3}\right)=c_{3} x_{3}+\hat{u}_{3}\left(x_{1}, x_{2}\right) .
\end{aligned}
$$

Substituting (3.57) into (3.54) $)_{1}$, one obtains $\hat{u}_{1}=x_{2} f\left(x_{3}\right)+g\left(x_{3}\right)$. Substituting this into $(3.54)_{2}$, one gets $x_{2} f^{\prime \prime}\left(x_{3}\right)+g^{\prime \prime}\left(x_{3}\right)=0$, which implies that $f^{\prime \prime}\left(x_{3}\right)=g^{\prime \prime}\left(x_{3}\right)=0$. This means that $u_{1}$ is the superposition of a homogeneous displacement field and the 1-parameter family of inhomogeneous displacements $a_{1} x_{2} x_{3}$. One finds similar expressions for the other two displacement components using (3.55) and (3.56). Therefore, we have the following characterization of the universal displacements.

Proposition 3.5. The universal displacements in an orthotropic linear elastic solid with planes of symmetry normal to the coordinate axes in a Cartesian coordinate system $\left(x_{1}, x_{2}, x_{3}\right)$ are the superposition of homogeneous displacement fields and the following 3-parameter inhomogeneous displacement field: $\left(a_{1} x_{2} x_{3}, a_{2} x_{1} x_{3}, a_{3} x_{1} x_{2}\right)$.

\subsection{Universal displacements in transversely isotropic linear elastic solids}

A transversely isotropic solid has an axis of symmetry such that planes normal to it are isotropy planes. Let us assume that the axis of transverse isotropy is the $x_{3}$-axis in the Cartesian coordinates $\left(x_{1}, x_{2}, x_{3}\right)$. A transversely isotropic solid has 5 independent elastic constants, and in the Cartesian coordinates $\left(x_{1}, x_{2}, x_{3}\right)$ the elasticity matrix has the following representation:

$$
\mathbf{c}=\left[\begin{array}{cccccc}
c_{11} & c_{12} & c_{13} & 0 & 0 & 0 \\
c_{12} & c_{11} & c_{13} & 0 & 0 & 0 \\
c_{13} & c_{13} & c_{33} & 0 & 0 & 0 \\
0 & 0 & 0 & c_{44} & 0 & 0 \\
0 & 0 & 0 & 0 & c_{44} & 0 \\
0 & 0 & 0 & 0 & 0 & \frac{1}{2}\left(c_{11}-c_{12}\right)
\end{array}\right]
$$


Navier's equations and arbitrariness of the five elastic constants result in the following PDEs for the universal displacements.

$$
\begin{aligned}
& \frac{\partial^{2} u_{3}}{\partial x_{1} \partial x_{3}}=\frac{\partial^{2} u_{3}}{\partial x_{2} \partial x_{3}}=\frac{\partial^{2} u_{3}}{\partial x_{3}^{2}}=0, \\
& \frac{\partial^{2} u_{3}}{\partial x_{1}^{2}}+\frac{\partial^{2} u_{3}}{\partial x_{2}^{2}}=0, \\
& \frac{\partial^{2} u_{1}}{\partial x_{3}^{2}}=\frac{\partial^{2} u_{2}}{\partial x_{3}^{2}}=0, \\
& \frac{\partial^{2} u_{1}}{\partial x_{1}^{2}}+\frac{\partial^{2} u_{1}}{\partial x_{2}^{2}}=\frac{\partial^{2} u_{2}}{\partial x_{1}^{2}}+\frac{\partial^{2} u_{2}}{\partial x_{2}^{2}}=0, \\
& \frac{\partial^{2} u_{1}}{\partial x_{2}^{2}}=\frac{\partial^{2} u_{2}}{\partial x_{1} \partial x_{2}}, \\
& \frac{\partial^{2} u_{2}}{\partial x_{1}^{2}}=\frac{\partial^{2} u_{1}}{\partial x_{1} \partial x_{2}}, \\
& \frac{\partial^{2} u_{1}}{\partial x_{1} \partial x_{3}}+\frac{\partial^{2} u_{2}}{\partial x_{2} \partial x_{3}}=0 .
\end{aligned}
$$

Note that the system of PDEs governing $u_{3}$ are decoupled from those governing $u_{1}$ and $u_{2}$. From (3.61) and (3.62) one concludes that

$$
u_{3}\left(x_{1}, x_{2}, x_{3}\right)=c_{3} x_{3}+\hat{u}_{3}\left(x_{1}, x_{2}\right), \quad \nabla^{2} \hat{u}_{3}=\frac{\partial^{2} \hat{u}_{3}}{\partial x_{1}^{2}}+\frac{\partial^{2} \hat{u}_{3}}{\partial x_{2}^{2}}=0
$$

From (3.63) one obtains

$$
u_{1}\left(x_{1}, x_{2}, x_{3}\right)=x_{3} f_{1}\left(x_{1}, x_{2}\right)+g_{1}\left(x_{1}, x_{2}\right), \quad u_{2}\left(x_{1}, x_{2}, x_{3}\right)=x_{3} f_{2}\left(x_{1}, x_{2}\right)+g_{2}\left(x_{1}, x_{2}\right) .
$$

(3.64) implies that

$$
\nabla^{2} f_{1}=\nabla^{2} f_{2}=\nabla^{2} g_{1}=\nabla^{2} g_{2}=0
$$

From (3.65)-(3.67) we have

$$
\begin{array}{ll}
\frac{\partial^{2} f_{1}}{\partial x_{2}^{2}}=\frac{\partial^{2} f_{2}}{\partial x_{1} \partial x_{2}}, & \frac{\partial^{2} f_{2}}{\partial x_{1}^{2}}=\frac{\partial^{2} f_{1}}{\partial x_{1} \partial x_{2}}, \\
\frac{\partial^{2} g_{1}}{\partial x_{2}^{2}}=\frac{\partial^{2} g_{2}}{\partial x_{1} \partial x_{2}}, & \frac{\partial^{2} g_{2}}{\partial x_{1}^{2}}=\frac{\partial^{2} g_{1}}{\partial x_{1} \partial x_{2}}, \\
\frac{\partial f_{1}}{\partial x_{1}}+\frac{\partial f_{2}}{\partial x_{2}}=0 . &
\end{array}
$$

Note that (3.71) can be rewritten as $\frac{\partial}{\partial x_{2}}\left(\frac{\partial f_{2}}{\partial x_{1}}-\frac{\partial f_{1}}{\partial x_{2}}\right)=\frac{\partial}{\partial x_{1}}\left(\frac{\partial f_{2}}{\partial x_{1}}-\frac{\partial f_{1}}{\partial x_{2}}\right)=0$, and hence, we have the following system of linear PDEs for $f_{1}$ and $f_{2}$ :

$$
\begin{aligned}
& \frac{\partial f_{1}}{\partial x_{1}}+\frac{\partial f_{2}}{\partial x_{2}}=0 \\
& \frac{\partial f_{1}}{\partial x_{2}}-\frac{\partial f_{2}}{\partial x_{1}}=c_{1} .
\end{aligned}
$$

The above are inhomogeneous Cauchy-Riemann equations, and hence, $f_{1}\left(x_{1}, x_{2}\right)=\frac{1}{2} c_{1} x_{2}+h_{1}\left(x_{1}, x_{2}\right), f_{2}\left(x_{1}, x_{2}\right)=$ $-\frac{1}{2} c_{1} x_{1}+h_{2}\left(x_{1}, x_{2}\right)$, where $\xi\left(x_{2}+i x_{1}\right)=h_{1}\left(x_{1}, x_{2}\right)+i h_{2}\left(x_{1}, x_{2}\right)$ is holomorphic. This implies that $(3.70)_{1}$ and $(3.70)_{2}$ are satisfied. From (3.72), we get $\frac{\partial g_{1}}{\partial x_{2}}-\frac{\partial g_{2}}{\partial x_{1}}=c_{2}$, and from $(3.70)_{3}$ and $(3.70)_{4}$, we find $\frac{\partial g_{1}}{\partial x_{1}}+\frac{\partial g_{2}}{\partial x_{2}}=c_{3}$. Therefore, $g_{1}\left(x_{1}, x_{2}\right)=\frac{1}{2} c_{3} x_{1}+\frac{1}{2} c_{2} x_{2}+k_{1}\left(x_{1}, x_{2}\right), g_{2}\left(x_{1}, x_{2}\right)=-\frac{1}{2} c_{2} x_{1}+\frac{1}{2} c_{3} x_{2}+k_{2}\left(x_{1}, x_{2}\right)$, where $\eta\left(x_{2}+i x_{1}\right)=$ $k_{1}\left(x_{1}, x_{2}\right)+i k_{2}\left(x_{1}, x_{2}\right)$ is holomorphic. Renaming the constants above we have the following characterization 
for the universal dispalcements.

Proposition 3.6. The universal displacements in a transversely isotropic linear elastic solid with the isotropy plane parallel to the $x_{1} x_{2}$-plane have the following form:

$$
\begin{aligned}
& u_{1}\left(x_{1}, x_{2}, x_{3}\right)=c_{1} x_{1}+c_{2} x_{2}+c x_{2} x_{3}+x_{3} h_{1}\left(x_{1}, x_{2}\right)+k_{1}\left(x_{1}, x_{2}\right), \\
& u_{2}\left(x_{1}, x_{2}, x_{3}\right)=-c_{2} x_{1}+c_{1} x_{2}-c x_{1} x_{3}+x_{3} h_{2}\left(x_{1}, x_{2}\right)+k_{2}\left(x_{1}, x_{2}\right), \\
& u_{3}\left(x_{1}, x_{2}, x_{3}\right)=c_{3} x_{3}+\hat{u}_{3}\left(x_{1}, x_{2}\right),
\end{aligned}
$$

where $\xi\left(x_{2}+i x_{1}\right)=h_{1}\left(x_{1}, x_{2}\right)+i h_{2}\left(x_{1}, x_{2}\right)$ and $\eta\left(x_{2}+i x_{1}\right)=k_{1}\left(x_{1}, x_{2}\right)+i k_{2}\left(x_{1}, x_{2}\right)$ are holomorphic, and $\hat{u}_{3}\left(x_{1}, x_{2}\right)$ is harmonic.

\subsection{Universal displacements in cubic linear elastic solids}

In a cubic solid at any point there are nine planes of symmetry with normals parallel to the edges and face diagonals of a cube. Consider a Cartesian coordinate system $\left(x_{1}, x_{2}, x_{3}\right)$ with coordinate lines parallel to the edges of the cube. In this coordinate system the matrix of elastic constants reads

$$
\mathbf{c}=\left[\begin{array}{cccccc}
c_{11} & c_{12} & c_{12} & 0 & 0 & 0 \\
c_{12} & c_{11} & c_{12} & 0 & 0 & 0 \\
c_{12} & c_{12} & c_{11} & 0 & 0 & 0 \\
0 & 0 & 0 & c_{44} & 0 & 0 \\
0 & 0 & 0 & 0 & c_{44} & 0 \\
0 & 0 & 0 & 0 & 0 & c_{44}
\end{array}\right] .
$$

Navier's equations and arbitrariness of the three elastic constants result in the following PDEs for the universal displacements.

$$
\begin{aligned}
& \frac{\partial^{2} u_{1}}{\partial x_{1}^{2}}=\frac{\partial^{2} u_{2}}{\partial x_{2}^{2}}=\frac{\partial^{2} u_{3}}{\partial x_{3}^{2}}=0 \\
& \frac{\partial^{2} u_{1}}{\partial x_{2}^{2}}+\frac{\partial^{2} u_{1}}{\partial x_{3}^{2}}=0 \\
& \frac{\partial^{2} u_{2}}{\partial x_{1}^{2}}+\frac{\partial^{2} u_{2}}{\partial x_{3}^{2}}=0 \\
& \frac{\partial^{2} u_{3}}{\partial x_{1}^{2}}+\frac{\partial^{2} u_{3}}{\partial x_{2}^{2}}=0 \\
& \frac{\partial^{2} u_{1}}{\partial x_{1} \partial x_{3}}+\frac{\partial^{2} u_{2}}{\partial x_{2} \partial x_{3}}=0 \\
& \frac{\partial^{2} u_{2}}{\partial x_{1} \partial x_{2}}+\frac{\partial^{2} u_{3}}{\partial x_{1} \partial x_{3}}=0 \\
& \frac{\partial^{2} u_{1}}{\partial x_{1} \partial x_{2}}+\frac{\partial^{2} u_{3}}{\partial x_{2} \partial x_{3}}=0
\end{aligned}
$$

From (3.79) we have

$$
\begin{aligned}
& u_{1}\left(x_{1}, x_{2}, x_{3}\right)=x_{1} f_{1}\left(x_{2}, x_{3}\right)+g_{1}\left(x_{2}, x_{3}\right), \\
& u_{2}\left(x_{1}, x_{2}, x_{3}\right)=x_{2} f_{2}\left(x_{1}, x_{3}\right)+g_{2}\left(x_{1}, x_{3}\right), \\
& u_{3}\left(x_{1}, x_{2}, x_{3}\right)=x_{3} f_{3}\left(x_{1}, x_{2}\right)+g_{3}\left(x_{1}, x_{2}\right) .
\end{aligned}
$$


(3.80)-(3.82) imply that $\nabla^{2} f_{a}=\nabla^{2} g_{a}=0, a=1,2,3$. From (3.80)-(3.82) one obtains

$$
\begin{aligned}
& \frac{\partial f_{1}}{\partial x_{3}}\left(x_{2}, x_{3}\right)+\frac{\partial f_{2}}{\partial x_{3}}\left(x_{1}, x_{3}\right)=0 \\
& \frac{\partial f_{2}}{\partial x_{1}}\left(x_{1}, x_{3}\right)+\frac{\partial f_{3}}{\partial x_{1}}\left(x_{1}, x_{2}\right)=0 \\
& \frac{\partial f_{1}}{\partial x_{2}}\left(x_{2}, x_{3}\right)+\frac{\partial f_{3}}{\partial x_{2}}\left(x_{1}, x_{2}\right)=0 .
\end{aligned}
$$

From (3.87) one concludes that $\frac{\partial f_{1}}{\partial x_{3}}$ and $\frac{\partial f_{2}}{\partial x_{3}}$ are only functions of $x_{3}$, and hence

$$
f_{1}\left(x_{2}, x_{3}\right)=F_{1}\left(x_{2}\right)+G_{1}\left(x_{3}\right), \quad f_{2}\left(x_{1}, x_{3}\right)=F_{2}\left(x_{1}\right)-G_{1}\left(x_{3}\right) .
$$

Substituting this into (3.88), we have

$$
f_{3}\left(x_{1}, x_{2}\right)=-F_{2}\left(x_{1}\right)+G_{3}\left(x_{2}\right) .
$$

Finally, substituting into (3.89) we get $F_{1}^{\prime}\left(x_{2}\right)+G_{3}^{\prime}\left(x_{2}\right)=0$, and hence, $G_{3}\left(x_{2}\right)=-F_{1}\left(x_{2}\right)+c$. In summary

$$
\begin{aligned}
& f_{1}\left(x_{2}, x_{3}\right)=\beta\left(x_{2}\right)+\gamma\left(x_{3}\right), \\
& f_{2}\left(x_{1}, x_{3}\right)=\alpha\left(x_{1}\right)-\gamma\left(x_{3}\right), \\
& f_{3}\left(x_{1}, x_{2}\right)=-\alpha\left(x_{1}\right)-\beta\left(x_{2}\right)+c .
\end{aligned}
$$

Knowing that $\nabla^{2} f_{1}=\nabla^{2} f_{2}=\nabla^{2} f_{3}=0$, one concludes that $\alpha^{\prime \prime}\left(x_{1}\right)=-\beta^{\prime \prime}\left(x_{2}\right)=\gamma^{\prime \prime}\left(x_{3}\right)=a$. Hence

$$
\begin{aligned}
& f_{1}\left(x_{2}, x_{3}\right)=\frac{a}{2}\left(x_{3}^{2}-x_{2}^{2}\right)+c_{1} x_{3}+b_{1} x_{2}+d_{1}, \\
& f_{2}\left(x_{1}, x_{3}\right)=\frac{a}{2}\left(x_{1}^{2}-x_{3}^{2}\right)+a_{1} x_{1}-c_{1} x_{3}+d_{2}, \\
& f_{3}\left(x_{1}, x_{2}\right)=\frac{a}{2}\left(x_{2}^{2}-x_{1}^{2}\right)-a_{1} x_{1}-b_{1} x_{2}+d_{3} .
\end{aligned}
$$

Therefore, the universal displacements have the representation (3.86) with $f_{a}$ given above and $g_{a}$ being harmonic functions.

Proposition 3.7. The universal displacements in a cubic linear elastic solid have the following form:

$$
\begin{aligned}
& u_{1}\left(x_{1}, x_{2}, x_{3}\right)=\frac{a}{2} x_{1}\left(x_{3}^{2}-x_{2}^{2}\right)+c_{1} x_{1} x_{3}+b_{1} x_{1} x_{2}+d_{1} x_{1}+g_{1}\left(x_{2}, x_{3}\right), \\
& u_{2}\left(x_{1}, x_{2}, x_{3}\right)=\frac{a}{2} x_{2}\left(x_{1}^{2}-x_{3}^{2}\right)+a_{1} x_{1} x_{2}-c_{1} x_{2} x_{3}+d_{2} x_{2}+g_{2}\left(x_{1}, x_{3}\right), \\
& u_{3}\left(x_{1}, x_{2}, x_{3}\right)=\frac{a}{2} x_{3}\left(x_{2}^{2}-x_{1}^{2}\right)-a_{1} x_{1} x_{3}-b_{1} x_{2} x_{3}+d_{3} x_{3}+g_{3}\left(x_{1}, x_{2}\right),
\end{aligned}
$$

where $g_{1}, g_{2}$, and $g_{3}$ are arbitrary harmonic functions.

\section{Conclusion}

We have obtained all the universal displacements in unconstrained linear elastic solids by requiring that the solutions of the Navier equations do not depend on the particular values of the elastic moduli. While our construction does not preclude the existence of other solutions, these solutions are the only solutions that can be maintained while varying the material parameters continuously. For instance, in the triclinic case, homogeneous solutions are the only possible solutions that can be achieved for various values of the elastic parameters as they are perturbed within an open set in the parameter space. It does not preclude the existence of other solutions, merely that any other solution cannot be maintained by changing the boundary tractions if the material parameters are perturbed. For instance, for triclinic solids nonhomogeneous solutions exist as soon as the tractions deviate from the homogeneous case, but their details depends on the material parameters, 
i.e., if we take two different triclinic solids and impose the same displacements at the boundaries, the internal displacements will differ unless the resultant solution is homogeneous.

In the present work, we have assumed a given symmetry class for the elastic material in order to find the corresponding universal solutions. We have also assumed that the directions of anisotropy are known and a particular basis to express the stiffness matrix is chosen. However, we note that in practice determining the symmetry class and these directions may be difficult. Similarly, determining whether two stiffness matrices define the same elastic material is problematic [Desmorat, Auffray, Desmorat, Kolev, and Olive, 2019]. We have also limited our study of anisotropic linear elastic systems to the compressible case but, a similar analysis can be done, in principle, for the incompressible anisotropic case and should yield interesting results.

The relationship between the material symmetry group and the class of solutions is expected but nevertheless is quite interesting. Indeed, we show that a general elastic triclinic material with no particular symmetry has no solution aside from the obvious homogeneous solution. As we expand the symmetry group, the space of solutions also expands accordingly. In the limiting case, isotropic elastic materials have a large space of solutions characterized by all constant-divergence harmonic displacement vector fields that explains the common use of isotropic linear elastic solids as models for materials. The small number of constants together with the zoo of associated solutions can be used to model a variety of possible configurations and to obtain elastic parameters that characterize particular materials.

Finally, it is interesting to note that in the geometric reduction of elasticity theory to rods, plates, and shells, it is common to obtain reduced problems that satisfy linear elasticity with a given geometry. For instance, in the reduction from three-dimensional elasticity to one-dimensional rod theory, the resolution of the stresses in the rod's cross-section requires the solution of a linear elasticity problem with orthotropic symmetry [Mora and Müller, 2003]. Not surprisingly, the solutions of this problem are exactly the ones that we obtain for that particular anisotropy class. Hence, we see that the universal displacements found for particular anisotropy classes are directly relevant to various problems of dimensional reductions in elasticity.

\section{Acknowledgments}

This research was supported by NSF - Grant No. CMMI 1130856, and ARO W911NF-16-1-0064 to Arash Yavari, and by the Engineering and Physical Sciences Research Council grant EP/R020205/1 to Alain Goriely.

\section{References}

D E Carlson. Dependence of linear elasticity solutions on the elastic constants. I. Dependence on Poisson's ratio in elastostatics. Journal of Elasticity, 1(2):145-151, 1971.

D E Carlson. Dependence of linear elasticity solutions on the elastic constants. II. Dependence on the shear modulus in elastostatics. Journal of Elasticity, 2(2):129-134, 1972.

P Chadwick, M Vianello, and S C Cowin. A new proof that the number of linear elastic symmetries is eight. Journal of the Mechanics and Physics of Solids, 49(11):2471-2492, 2001.

S C Cowin and S B Doty. Tissue Mechanics. Springer Science \& Business Media, 2007.

S C Cowin and M M Mehrabadi. Anisotropic symmetries of linear elasticity. Applied Mechanics Reviews, 48 (5):247-285, 1995.

R Desmorat, N Auffray, B Desmorat, B Kolev, and M Olive. Generic separating sets for three-dimensional elasticity tensors. Proceedings of the Royal Society A, 475(2226):20190056, 2019.

J L Ericksen. Deformations possible in every isotropic, incompressible, perfectly elastic body. Zeitschrift für Angewandte Mathematik und Physik (ZAMP), 5(6):466-489, 1954.

J L Ericksen. Deformations possible in every compressible, isotropic, perfectly elastic material. Journal of Mathematics and Physics, 34(1-4):126-128, 1955. 
R L Fosdick. Remarks on Compatibility. Modern Developments in the Mechanics of Continua, pages 109-127, 1966.

A. Goriely. The Mathematics and Mechanics of Biological Growth. Springer Verlag, New York, 2017.

M. E. Gurtin. The linear theory of elasticity. In Handbuch der Physik, Band VIa/2. Springer-Verlag, Berlin, 1972.

W W Klingbeil and R T Shield. On a class of solutions in plane finite elasticity. Zeitschrift für angewandte Mathematik und Physik ZAMP, 17(4):489-511, 1966.

R I McLachlan and G R W Quispel. Splitting methods. Acta Numerica, 11:341-434, 2002.

M G Mora and S Müller. Derivation of the nonlinear bending-torsion theory for inextensible rods $\Gamma$-convergence. Calculus of Variations and Partial Differential Equations, 18(3):287-305, 2003.

R S Rivlin. Large elastic deformations of isotropic materials IV. Further developments of the general theory. Philosophical Transactions of the Royal Society of London A, 241(835):379-397, 1948.

R S Rivlin. Large elastic deformations of isotropic materials. V. the problem of flexure. Proceedings of the Royal Society of London A, 195(1043):463-473, 1949a.

R S Rivlin. A note on the torsion of an incompressible highly elastic cylinder. In Mathematical Proceedings of the Cambridge Philosophical Society, volume 45, pages 485-487. Cambridge University Press, 1949 b.

G Saccomandi. Universal results in finite elasticity. In Y. B. Fu and R. W. Ogden, editors, Non-Linear Elasticity: Theory and Applications, chapter 3, pages 97-134. Cambridge University Press, 2001.

M Singh and A C Pipkin. Note on Ericksen's problem. Zeitschrift für angewandte Mathematik und Physik ZAMP, 16(5):706-709, 1965.

E B Tadmor, R E Miller, and R S Elliott. Continuum Mechanics and Thermodynamics: From Fundamental Concepts to Governing Equations. Cambridge University Press, 2012.

T C T Ting. Generalized Cowin-Mehrabadi theorems and a direct proof that the number of linear elastic symmetries is eight. International Journal of Solids and Structures, 40(25):7129-7142, 2003.

C Truesdell. The mechanical foundations of elasticity and fluid dynamics. Journal of Rational Mechanics and Analysis, 1:125-300, 1952.

C Truesdell. The Elements of Continuum Mechanics. Springer-Verlag, 1966.

C. Truesdell and W. Noll. The Non-Linear Field Theories of Mechanics. The non-linear field theories of mechanics. Springer, $3^{\text {rd }}$ edition, 2004. ISBN 9783540027799.

A Yavari and A Goriely. The anelastic Ericksen problem: Universal eigenstrains and deformations in compressible isotropic elastic solids. Proceedings of the Royal Society A, 472(2196):20160690, 2016. 